\title{
Which patients with locally advanced pancreatic cancer treated with induction chemotherapy are most likely to benefit from post-induction chemoradiotherapy?
}

\author{
Sophie Otter, Irene Chong, Ria Kalaitzaki, Diana Tait
}

\section{ABSTRACT}

Aims: The role of concomitant chemotherapy with radiotherapy (CRT) in locally advanced pancreatic cancer (LAPC) is controversial. The aim of this study was to report the outcomes of patients with LAPC treated with CRT over a 10year period within a single institution and to identify those patients who derived the most benefit. Methods: Patients with LAPC who received radical radiotherapy $(\geq 45 \mathrm{~Gy})$ between January 2004 - October 2014 were identified. The Electronic Patient Record was reviewed to collect data regarding staging, treatment, response and outcome. The Kaplan-Meier and Cox regression methods were used to analyse survival outcomes and compare survival rates between groups. Results: 138 patients were identified. Patients who had a response on imaging after induction chemotherapy had a median OS of 17.4 months compared to 10.3 months in non-responders (HR $0.55,95 \%$ CI $0.35-0.87, \mathrm{p}=0.01)$. At three months post-radiotherapy, patients who had achieved a response on CT had a median OS of 56 months compared to 10.7 months (HR 0.28, 95\% CI 0.12$0.65, p=0.003$ ). However, a reduction in CA199 prior to radiotherapy was not significantly associated with progression free survival (PFS)

Sophie Otter ${ }^{1}$, Irene Chong ${ }^{2}$, Ria Kalaitzaki ${ }^{3}$, Diana Tait ${ }^{2}$

Affiliations: ${ }^{1}$ Clinical Research Fellow, Royal Marsden Hospital, UK; ${ }^{2}$ Consultant Clinical Oncologist, Royal, Marsden, Hospital, UK; ${ }^{3}$ Senior Statistician, Royal Marsden Hospital, UK.

Corresponding Author: Dr. Diana Tait, Royal Marsden Hospital, Downs Road, Sutton, UK, SM2 5PT; Email: diana. tait@rmh.nhs.uk

Received: 22 December 2017

Accepted: 23 February 2018

Published: 06 March 2018 or Overall survival (OS). Patients with a response in CA19-9 levels at 3-months post-radiotherapy compared to baseline had an OS of 19.1 months compared to 10.5 months in non-responders (HR $0.42,95 \%$ CI $0.26-0.68, p<0.001)$. Conclusion: Patients with LAPC who responded to chemotherapy on imaging prior to radiotherapy had improved PFS and OS than non-responders and therefore appeared to benefit the most from CRT. A decrease in CA19-9 prior to radiotherapy was not associated with improved survival and proved less useful for patient selection for CRT.

Keywords: Chemoradiotherapy, Pancreatic cancer, PET-CT

\section{How to cite this article}

Otter S, Chong I, Kalaitzaki R, Tait D. Which patients with locally advanced pancreatic cancer treated with induction chemotherapy are most likely to benefit from post-induction chemoradiotherapy? Int J Hepatobiliary Pancreat Dis 2018;8:100077Z04SO2018.

Article ID: $100077 \mathrm{Zo4SO} 2018$

$* * * * * * * * *$

doi:10.5348/100077Zo4SO2018OA

\section{INTRODUCTION}

Pancreatic cancer is the tenth most common cancer in the UK. In 2013, there were 9,408 new cases of pancreatic cancer. The 10-year survival in England and Wales from 2010-2011 was <1\% [1]. Patients with locally advanced disease have an overall survival of 6-11 months [2]. 


\section{EDORIUM Journals}

In 1981, the Gastrointestinal Tumor Study Group showed that radiotherapy with bolus $5 \mathrm{FU}$ was superior to radiotherapy alone with a one year survival of $40 \% \mathrm{v} .10 \%$ [3]. However, there is still some controversy about the role of CRT (concomitant chemotherapy with radiotherapy) versus chemotherapy alone in the treatment of locally advanced pancreatic cancer (LAPC), largely due to differences in chemotherapy regimens, scheduling and duration.

A phase three randomised controlled trial (LAPo7) compared chemotherapy alone (gemcitabine +/erlotinib) for six months with induction chemotherapy (gemcitabine +/- erlotinib) for four months followed by chemoradiotherapy (54Gy in 30\# with capecitabine) [4]. There was no significant difference in overall survival (OS) between the chemotherapy and the chemoradiotherapy arms. The rate of local recurrence was lower in patients receiving chemoradiotherapy $32 \%$ vs $46 \%, p=0.03$ ) and there was no increase in grade 3 or 4 toxicities. However, it should be noted that the induction chemotherapy was only for four months and that patients received gemcitabine alone rather than a doublet or triplet chemotherapy regimen.

A meta-analysis in 2007 identified two randomised controlled trials which compared CRT followed by chemotherapy with chemotherapy alone where no improvement in OS in the CRT arm was observed (two trials, 134 patients, HR 0.79; 95\% CI 0.32-1.95) [5]. A study undertaken by Chauffert et al. revealed a survival disadvantage for CRT compared to chemotherapy but incorporated a regimen of cisplatin and $5 \mathrm{FU}$ in combination with radiotherapy that was poorly tolerated and which would not be considered standard treatment [6].

However, there are other studies which suggest a positive role for radiotherapy in patients with LAPC $[7,8]$. A phase two randomised trial of patients with LAPC $(n=74)$ compared six cycles of gemcitabine with chemoradiotherapy (weekly gemcitabine, 50.4 Gy in $28 \#$ ). There were more grade four and five toxicities (41\% v 9\%) in the CRT arm but grade 3 and 4 toxicities were similar. Survival in the chemotherapy arm was 9.2 months compared to 11.1 months in the chemoradiotherapy arm $(\mathrm{p}=0.017)[7]$.

Furthermore, three retrospective studies have reported an improvement in OS. Choi et al. reported that patients with unresectable LAPC who received concurrent CRT had a significantly improved OS of 15.4 months compared to 9.3 months in patients who had chemotherapy alone $(\mathrm{p}=0.011)$. HR for OS was $0.536(\mathrm{p}=0.003)$ [9]. Huang et al. also retrospectively identified a survival benefit for chemoradiotherapy over chemotherapy alone (OS 14.6 months v 8.1 months, $\mathrm{p}=0.001$ ) [10].

The SCALOP trial was a phase 2 trial randomising patients to chemoradiotherapy with either gemcitabine or capecitabine after 16 weeks of induction gemcitabine and capecitabine chemotherapy. There was no control arm of continuing with chemotherapy alone but the results showed that the capecitabine regimen had an improvement in median OS of three months (although the HR was not significant) and was less toxic than the gemcitabine arm $[11,12]$. SCALOP2 is currently recruiting and is investigating both dose escalation of radiotherapy to $60 \mathrm{~Gy}$ in $30 \#$ and also the addition of nelfinavir (a protease inhibitor which inhibits the Akt/PKB pathway) to chemoradiotherapy [13]. The trial design includes a chemotherapy only arm and also an observation arm. The combination of novel drugs with radiotherapy and dose escalation will hopefully lead to further improved local control and overall survival.

From the available literature, with conflicting results and an inability to demonstrate an overall role for CRT in LAPC, the benefit of CRT may be in a select group of patients and therefore selection criteria for CRT need to be better defined. We report the outcome data of 138 consecutive patients receiving CRT at a single institution. In addition, we focus on the utility of imaging response assessments after neo-adjuvant chemotherapy and changes in CA19-9 levels in an attempt to identify patients who had improved survival following chemoradiotherapy.

\section{MATERIALS AND METHODS}

Patients with LAPC who, after induction chemotherapy, were treated with radiotherapy at a dose of $45 \mathrm{~Gy}$ or greater at a single institution between January 2004 - October 2014 were identified. Their Electronic Patient Record was reviewed to collect data regarding demographics, staging investigations, radiotherapy and chemotherapy details, responsetotreatmentand outcome. Responders on imaging (PET-CT or CT) were defined as those patients who had either complete response (CR) or partial response (PR) using Response Evaluation Criteria in Solid Tumors (RECIST) criteria. Overall survival (OS) was defined as the time from radiotherapy start date to death from any cause whilst progression free survival (PFS) was measured from radiotherapy start date to date of progression or death from any cause. The start of radiotherapy was chosen as it was a more definitive time point particularly as some patients had been diagnosed at other institutions and may have received their induction chemotherapy elsewhere making the date of diagnosis more difficult to define. Patients without an event were censored at last follow up. The Kaplan-Meier method was used to summarise the survival estimates whilst Cox regression method was used to compare the survival rates between groups. CT imaging and CA 19-9 were routinely performed for staging and response assessment. PET was not used routinely for staging before 2008 and has not been a routine part of response assessment at our institution. 


\section{EDORIUM Journals}

Int J Hepatobiliary Pancreat Dis 2018;8:100077Z04SO2018. www.ijhpd.com

\section{RESULTS}

\section{Patient demographics and tumour staging}

Between January 2004 - October 2014, 138 patients with pancreatic cancer were treated with radical radiotherapy (doses $>45$ Gy). Baseline characteristics are displayed in Table 1. Although there was no formal TNM staging documented on radiology reports for staging investigations or in the MDT (multidisciplinary team) summary for $51.5 \%(71 / 138)$ patients, the majority of these cases were documented as locally advanced disease by specialist radiologists who defined this as encasement of vessels or other local spread contraindicating surgery. The PFS for the whole cohort was six months (IQR 3.710.3 months) and OS was 12 months (IQR 6.6-22.7 months).

\section{Treatment details}

97.1\% (134/138) of patients received chemotherapy prior to radiotherapy (Table 2). Although the four patients who did not receive induction chemotherapy had ECOG performance statuses of 1-2, they were aged 72-86 and therefore may have had other comorbidities that precluded the delivery of systemic treatment. The majority of patients $(80 / 134,59.7 \%)$ received between 4-6 months of chemotherapy. A minority (6/134, 4.4\%) switched from one chemotherapy regimen to another due to progression or toxicity.

$84.8 \%(117 / 138)$ of patients received concurrent CRT (Table 2). Of the patients receiving concurrent CRT, 87.2\% (102/117) completed the full course of concurrent chemotherapy. $7.2 \%$ of patients (10/138) had their radiotherapy terminated early. 91.3\% (126/138) of patients tolerated radiotherapy with or without chemotherapy well and without any grade 3 or 4 toxicities.

\section{PET scans}

$67 \%$ of patients had a PET-CT scan at presentation. This was in addition to a staging CT scan which was performed on all patients. The majority of patients who were not staged with a PET-CT scan were treated prior to 2008 when PET-CT was less readily available. Following induction chemotherapy, 21 patients (15.7\%) had a further PET-CT scan. In 10 of those patients the PET-CT and CT results were consistent, 8 patients had a greater response on PET-CT (for example complete metabolic response on PET-CT compared to PR on CT), 1 patient had PR on CT but stable disease on PET-CT and 2 patients did not have a corresponding CT result that was available. At relapse, 32/122 (26.2\%) had PET-CT scans.

\section{Response on imaging and Survival Outcomes}

The schema for the treatment and imaging schedule followed is outlined at the top of Table 3. CT response to induction chemotherapy is shown, as is the CT response at 3 months post-completion of chemoradiation. Of the 33 patients that progressed at the 3 month post radiotherapy imaging point, 19 (57.6\%) had distant metastases, 8 (24.2\%) had local progression only and 6 (18.2\%) had both local and distant progression.

Those that responded on imaging criteria (CR or PR on PET-CT or CT) after induction chemotherapy had a median PFS of 7.5 months compared with 5.3 months for those assessed as non-responders (Figure 1 (A), HR 0.72, 95\% CI 0.48-1.07, $\mathrm{p}=0.11$ ). Responders had a median OS of 17.4 months whilst non-responders had a median OS of 10.3 months (Figure 1 (B), HR 0.55,95\% CI 0.35-0.87, $\mathrm{p}=0.01)$.

Of the 19 patients who relapsed, 16 went on to receive second line chemotherapy or radiofrequency ablation (Gemcitabine / capecitabine $=9$, Folfirinox $=3$, Gemcitabine / abraxane=1, Folfox=1, Gemcitabine / oxaliplatin $=1$ and $\mathrm{RFA}=1$ ).

The PFS and OS results for the patient groups classified as responders and non-responders at 3 months post completion of chemo-RT are shown in (Figure 2A and $\mathrm{B})$.

\section{CA19-9 Response and survival outcomes}

$89(71.8 \%)$ patients had a decrease in CA19-9 from baseline to pre-CRT and 35 (28.2\%) had no change or an increase (the remaining patients had blood tests results missing as they were performed at other hospitals). 25 patients had a decrease in CA19-9 from pre-CRT to 3 months post-CRT time points and 75 had either no change or an increase.

Neither PFS nor OS was significantly correlated with a fall in CA19-9 after induction chemotherapy and before the start of CRT. Comparing CA19-9 responders with non-responders, PFS was 6.7 months compared with 4.1 months and median OS 13 months compared with 9.6 months. However, there was a significant improvement in PFS in patients showing a decrease in CA19-9 from baseline to 1 month post-radiotherapy, 7.5 months compared to 3.6 months, HR 0.43 (95\% CI 0.27-0.69, $\mathrm{p}<0.001)$. Similarly with OS, those with any decrease had a median OS of 14.4 months compared to 7.3 months in those without, HR 0.58 (95\% CI 0.35-0.95, p=0.03). This effect persisted at the 3 months post radiotherapy assessment with a PFS of 9 months compared to 5.3 months, HR 0.43 (95\% CI 0.27-0.69 p<0.001) and OS 19.1 months compared to 10.5 months, HR 0.42 (95\% CI o.26-0.68), $\mathrm{p}<0.001)$.

CA19-9 level pre-radiotherapy compared with those at 3 months post radiotherapy showed a similar effect. Median PFS was 11.7 months in those with any decrease and 5.7 months in those without, HR 0.37 (95\% CI 0.22-0.63, $\mathrm{p}<0.001)$. The median OS figure for the two groups was non-significant with figures of 20.6 months compared with 11.0 months, HR 0.44 (95\% CI 0.25-0.77, $\mathrm{p}=0.01)$. 


\section{EDORiUM Journals}

Int J Hepatobiliary Pancreat Dis 2018;8:100077Z04SO2018.

www.ijhpd.com

Table 1: Baseline patient and treatment characteristics

\begin{tabular}{|c|c|c|}
\hline Characteristics & Category & Frequency, $n=138(\%)$ \\
\hline \multirow[t]{2}{*}{ Gender (\%) } & Male & $75(54 \%)$ \\
\hline & Female & $63(46 \%)$ \\
\hline \multirow[t]{5}{*}{ Age (\%) } & $40-49$ & $8(5.8 \%)$ \\
\hline & $50-59$ & $33(23.9 \%)$ \\
\hline & $60-69$ & $58(42.0 \%)$ \\
\hline & $70-79$ & $35(25.4 \%)$ \\
\hline & $80-89$ & $4(2.9 \%)$ \\
\hline \multirow[t]{5}{*}{ Performance status (\%) } & o & $32(23.2 \%)$ \\
\hline & 1 & $58(42.0 \%)$ \\
\hline & 2 & $10(7.2 \%)$ \\
\hline & 3 & $1(0.7 \%)$ \\
\hline & Not documented & $37(26.8 \%)$ \\
\hline \multirow[t]{3}{*}{ T staging (\%) } & $\mathrm{T} 2$ & $5(3.6 \%)$ \\
\hline & $\mathrm{T} 3$ & $13(9.4 \%)$ \\
\hline & $\mathrm{T} 4$ & $46(33.3 \%)$ \\
\hline \multirow[t]{3}{*}{ N staging (\%) } & No & $33(23.9 \%)$ \\
\hline & $\mathrm{N} 1$ & $29(21 \%)$ \\
\hline & N2 & $2(1.4 \%)$ \\
\hline Locally advanced & No formal TNM staging & $71(51.5 \%)$ \\
\hline Metastatic (\%) & M1 & $3(2.2 \%)$ \\
\hline CA $19-9$ at presentation & 190.5 (median) & $41-905$ (range) \\
\hline \multirow[t]{2}{*}{ PET at presentation } & Yes & $92(66.7 \%)$ \\
\hline & No & $46(33 \cdot 3 \%)$ \\
\hline \multirow[t]{4}{*}{ Radiotherapy dose } & $45 \mathrm{~Gy}$ in $25^{\#}$ & $2(1.4 \%)$ \\
\hline & $50.4 \mathrm{~Gy}$ in $28 \#$ & $44(31.9 \%)$ \\
\hline & $54 \mathrm{~Gy}$ in $30 \#$ & $89(64.5 \%)$ \\
\hline & 60 Gy in $30 \#$ & $3(2.2)$ \\
\hline
\end{tabular}

Table 2: Induction and concurrent chemotherapy rates

\begin{tabular}{|c|c|c|c|c|}
\hline Type of chemotherapy & $\begin{array}{l}\text { Chemotherapy } \\
\text { received? }\end{array}$ & Frequency (\%) & Regimen & Frequency \\
\hline \multirow[t]{2}{*}{ Induction chemotherapy } & Yes & $134(97.1 \%)$ & $\begin{array}{c}\text { Gemcitabine } \\
\text { Gemcitabine / } \\
\text { capecitabine } \\
\text { Trial } \\
\text { FOLFIRINOX } \\
\text { Other }\end{array}$ & $\begin{array}{c}14(10.4 \%) \\
63(47 \%) \\
33(24.6 \%) \\
17(12.7 \%) \\
7(5.2 \%)\end{array}$ \\
\hline & No & $4(2.9 \%)$ & & \\
\hline \multirow[t]{2}{*}{ Concurrent chemotherapy } & Yes & $117(84.8 \%)$ & $\begin{array}{l}\text { Capecitabine } \\
\text { Other }\end{array}$ & $\begin{array}{c}110(94 \%) \\
7(6 \%)\end{array}$ \\
\hline & No & $21(15.2 \%)$ & & \\
\hline
\end{tabular}




\section{EDORIUM Journals}

Int J Hepatobiliary Pancreat Dis 2018;8:100077Z04SO2018.

www.ijhpd.com

\section{Surgery}

16 of the 138 patients in this series (11.6\%) proceeded to radical surgery following completion of chemoradiation. eight received Folfirinox as induction chemotherapy, six received gemcitabine / capecitabine and the remaining patients received gemcitabine / oxaliplatin or trial regimens.

The PFS for patients who proceeded to surgery $(16 / 138,11.6 \%)$ was 20.3 months compared to 5.3 months for those who did not (HR $0.21,95 \%$ CI 0.10 to 0.43 , $\mathrm{p}<0.001$ ) whilst OS was 43.5 months and 10.7 months respectively (HR $0.21,95 \%$ CI 0.09 to $0.49, \mathrm{p}<0.001$ (Figure 2 (C) and (D)).

\section{Patterns of recurrence following CRT}

Relapse, at any time point, was with distant metastases in $48.6 \%(67 / 138)$ of patients, local recurrence in $23.9 \%$ (33/138) and both distant and local disease in 8\% (11/138). No relapse was documented for 19/138 patients (13.8\%). The remaining $5.8 \%(8 / 138)$ were lost to follow up, were presumed to have metastases but were never imaged or had metastases found at surgery. Sites of relapse in order of frequency were pancreas $22.5 \%(31 / 138)$, liver $19.6 \%$ (27/138) and peritoneum $17.4 \%$ (24/138). Of those with documented relapse, 53.8\% (64/119) went on to have further treatment with either chemotherapy, radiofrequency ablation or palliative radiotherapy.

\section{DISCUSSION}

The incidence of pancreatic cancer is predicted to increase due to an increase in risk factors such as obesity and diabetes. This, coupled with the poor prognosis, means that it is a significant cause of morbidity and mortality worldwide. However, intriguingly there

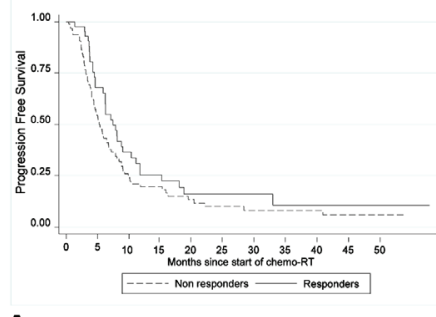

A

Figure 1: PFS (A) and OS (B) for responders and non-responders to induction chemotherapy assessed on imaging. PFS and OS are measured from the start of radiotherapy. Doublet / triplet chemotherapy included gemcitabine / capecitabine, FOLFIRNOX and trial regimens.
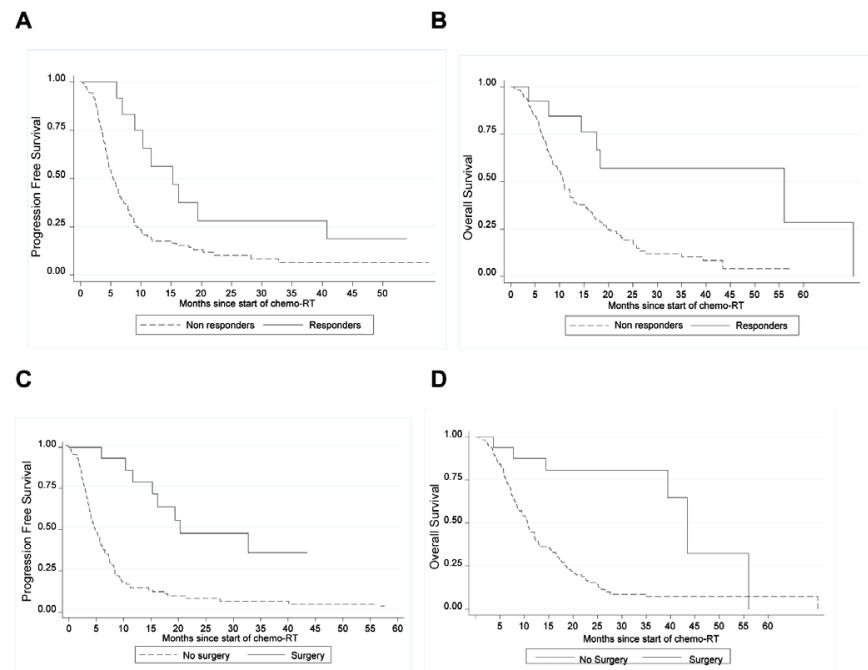

Figure 2: PFS (A) and OS (B) according to imaging response three months post chemoradiotherapy. PFS (C) and OS (D) according to whether patients proceeded to surgery.

Table 3: Schema of treatment and imaging scheduling showing response to induction chemotherapy (compared to CT at diagnosis) and response to chemoradiotherapy (compared to CT pre-radiotherapy).

\begin{tabular}{|c|c|c|c|c|}
\hline $\begin{array}{l}\text { Baseline staging and } \\
\text { imaging }\end{array}$ & $\begin{array}{c}\text { Induction } \\
\text { chemotherapy } \\
\longrightarrow\end{array}$ & $\begin{array}{c}\text { Response to induction } \\
\text { chemotherapy assessed by } \\
\text { CT }\end{array}$ & $\stackrel{\text { (Chemo)-RT }}{\longrightarrow}$ & $\begin{array}{l}\text { CT Response } 3 \text { month } \\
\text { post-(Chemo)-RT } \\
\longrightarrow\end{array}$ \\
\hline CT response & $\mathrm{n}=134$ & & $\mathrm{n}=138$ & \\
\hline Complete Response & & o (o\%) & & $3(2.1 \%)$ \\
\hline Partial Response & & $38(28.4 \%)$ & & $10(7 \cdot 3 \%)$ \\
\hline Stable Disease & & $76(56.7 \%)$ & & $68(49.3 \%)$ \\
\hline Progressive Disease & & $14(10.4 \%)$ & & $33(23.9 \%)$ \\
\hline $\begin{array}{l}\text { Results not available/ } \\
\text { applicable }\end{array}$ & & $6(4.5 \%)$ & & $24(17.4 \%)$ \\
\hline
\end{tabular}




\section{EDORIUM Journals}

heterogeneous disease presenting challenges in selection for treatment approaches.

The role of chemoradiotherapy remains controversial in the management of locally advanced pancreatic cancer but there is a subset of patients who appear to benefit and the aim of this retrospective study was to try to identify those patients. These patients may have an improved prognosis due to the molecular profile of their disease that could confer a more indolent phenotype facilitating the successful delivery of localised treatment. In this series, the patients that had particularly good outcomes after chemoradiotherapy were the 13 patients that showed an imaging response 3-month post-radiotherapy (median OS 56 months). 8 of these 13 went on to have a Whipple's procedure. Therefore, the role of CRT may be particularly important in downstaging a small group of LAPC patients thereby allowing them to proceed to surgery. However, the next conundrum is how to identify these patients upfront that are initially felt to be locally advanced but over the course of chemotherapy and CRT become operable.

It may be that surgery should be the ultimate goal of using chemoradiotherapy in LAPC as other studies have also demonstrated better survival in patients able to undergo surgery following chemoradiation. A multicentre UK retrospective study reported improved survival (27 m $\mathrm{v} 6 \mathrm{~m}, \mathrm{p}=0.023$ ) in patients undergoing resection [14]. However, surgery is not an option for the majority of patients as was seen in a further retrospective series in which only 1 of 54 patients proceeded to resection [15]. This latter study was small but there is a large systematic review of 111 studies which has shown that approximately one third of patients deemed to have unresectable disease at diagnosis, became resectable after neoadjuvant treatment and had comparable survival to those patients who had been deemed to be resectable upfront [16].

The rationale for intensifying local treatment, such as surgery or radiotherapy is supported in our study with $24 \%$ of relapsing patients having local disease only. As far as radiotherapy is concerned, there are a number of approaches that might be used to reduce local recurrence rates and dose escalation is now a reality using more advanced radiotherapy techniques. For example, patients treated with higher doses (BED > 70 Gy) achieved with intensity modulated radiotherapy (IMRT) and simultaneous integrated boost, have a superior OS (17.8 vs 15.0 months, $\mathrm{p}=0.03$ ) [17]. IMRT together with image guided radiotherapy (IGRT) may allow greater certainty in terms of tumour localization and accuracy of delivery, and therefore permit a safe reduction in radiotherapy planning margins [18].

The precision of tumor delineation also needs to be taken into consideration as there is considerable variability of tumour outlining between oncologists even in trials such as the SCALOP trial where there was a clearly defined protocol [19]. Other imaging modalities such as PET-CT may allow more accurate target delineation and therefore improve local control [20]. Pre- chemoradiotherapy PET-CT parameters (SUVmax <6.2) has also been shown to predict patients that are less likely to metastasise and therefore may benefit most from local treatment intensification [21].

Treatment delivery may also be further improved with the introduction of the MR linear accelerator. This, combined with diagnostic and planning MRs, may increase target volume and treatment delivery certainty. Other approaches that allow radiotherapy dose escalation include Stereotactic body radiotherapy (SBRT) $[18,19]$. SBRT in $3-5$ fractions either preceding or following systemic chemotherapy has been shown in non-randomised studies to improve local control rates with an acceptable toxicity profile [22, 23].

Although there was a significant local only relapse rate (24\%) in our series, as expected, the majority of patients relapsed with systemic disease. This emphasises the importance of considering this disease as a systemic problem and the need to also look at ways of improving systemic therapy. For example, there may be a role for maintenance chemotherapy. This approach has already shown promise in patients who have had their pancreatic cancer resected and who had an improved survival, compared with adjuvant chemotherapy alone, by the introduction of maintenance chemotherapy [22]. As yet, there are no phase III trials to provide definitive evidence for this approach and, similarly, other strategies to reduce distant metastases might involve novel drugs in combination with conventional chemotherapy.

Overall, our results confirm that imaging response after induction chemotherapy and post chemoradiation, predicts for improved PFS and OS. High resolution imaging, including MRI, may provide an even finer handle on the impact of treatment on tumour structure and function. However, the presented results on the role of CA19-9 don't support the use of marker-response as a reliable means of identifying good outcomes, at least after induction chemotherapy. The better outcomes for patients demonstrating a fall in CA19-9 after chemoradiation doesn't help in selecting patients for Chemoradiotherapy but may help in discussions on prognosis after treatment.

Although this study has limitations including its retrospective nature and limited size, it represents a valuable resource describing our experience in treating patients with locally advanced pancreatic cancer over the last decade. There has been a consistent approach of aiming to deliver 6 months of neoadjuvant chemotherapy prior to chemoradiation and the present population provides a significant sample size for this strategy. Furthermore, the reported population is representative of the LAPC cancer population as a whole, rather than just those selected for trial inclusion. However, in order to fully determine the contribution of chemoradiotherapy to the outcome of these patients, a control arm of patients who received no chemoradiotherapy would be needed. This would therefore determine whether the patients who responded well to induction chemotherapy would have 


\section{EDORIUM Journals}

continued to have an improved survival with or without CRT.

\section{CONCLUSION}

In this study, we observed that patients who responded to induction chemotherapy on imaging, prior to radiotherapy, achieved an improved PFS and OS compared with non-responders and appeared to derive the greatest benefit from CRT. It seems reasonable, therefore, to suggest that radiological response following induction chemotherapy could be useful in the selection of patients for subsequent CRT. Although we cannot definitively conclude that the addition of CRT contributed to these improved outcomes, it would be worthwhile to prospectively evaluate the utility of imaging response following induction chemotherapy as a radiological biomarker to stratify patients for subsequent local therapy.

\section{REFERENCES}

1. http://www.cancerresearchuk.org/healthprofessional/cancer-statistics/statistics-by-cancertype/pancreatic-cancer/survival-heading-Three

2. Amikura K, Kobari M, Matsuno S. The time of occurrence of liver metastasis in carcinoma of the pancreas. Int J Pancreatol 1995 Apr;17(2):139-46.

3. Moertel CG, Frytak S, Hahn RG, et al. Therapy of locally unresectable pancreatic carcinoma: A randomized comparison of high dose (6000 rads) radiation alone, moderate dose radiation (4000 rads +5 -fluorouracil), and high dose radiation + 5-fluorouracil: The gastrointestinal tumor study group. Cancer 1981 Oct 15;48(8):1705-10.

4. Hammel P, Huguet F, van Laethem JL, et al. Effect of chemoradiotherapy vs chemotherapy on purvival in patients with locally advanced pancreatic cancer controlled after 4 months of gemcitabine with or without erlotinib: The LAPO7 randomized clinical trial. JAMA 2016 May 3;315(17):1844-53.

5. Sultana A, Tudur Smith C, Cunningham D, et al. Systematic review, including meta-analyses, on the management of locally advanced pancreatic cancer using radiation/combined modality therapy. $\mathrm{Br} \mathrm{J}$ Cancer 2007 Apr 23;96(8):1183-90.

6. Chauffert B, Mornex F, Bonnetain F, et al. Phase III trial comparing intensive induction chemoradiotherapy (6o Gy, infusional 5-FU and intermittent cisplatin) followed by maintenance gemcitabine with gemcitabine alone for locally advanced unresectable pancreatic cancer. Definitive results of the 2000-01 FFCD/SFRO study. Ann Oncol 2008 Sep;19(9):15929.

7. Loehrer PJ Sr, Feng Y, Cardenes H, et al. Gemcitabine alone versus gemcitabine plus radiotherapy in patients with locally advanced pancreatic cancer: An eastern cooperative oncology group trial. J Clin Oncol 2011 Nov 1;29(31):4105-12.
8. Huguet $\mathrm{F}$, André $\mathrm{T}$, Hammel $\mathrm{P}$, et al. Impact of chemoradiotherapy after disease control with chemotherapy in locally advanced pancreatic adenocarcinoma in GERCOR phase II and III studies. J Clin Oncol 2007 Jan 20;25(3):326-31.

9. Choi Y, Oh DY, Kim K, et al. Concurrent chemoradiotherapy versus chemotherapy alone for unresectable locally advanced pancreatic cancer: A retrospective cohort study. Cancer Res Treat 2016 Jul;48(3):1045-55.

10. Huang WK, Kuo YC, Tsang NM, et al. Concurrent chemoradiotherapy with or without induction chemotherapy versus chemotherapy alone in patients with locally advanced pancreatic cancer. Anticancer Res 2014 Nov;34(11):67553-61.

11. Mukherjee S, Hurt CN, Bridgewater J, et al. Gemcitabine-based or capecitabine-based chemoradiotherapy for locally advanced pancreatic cancer (SCALOP): A multicentre, randomised, phase 2 trial. Lancet Oncol 2013 Apr;14(4):317-26.

12. Hurt CN, Falk S, Crosby $\mathrm{T}$, et al. Long-term results and recurrence patterns from SCALOP: A phase II randomised trial of gemcitabine- or capecitabinebased chemoradiation for locally advanced pancreatic cancer. Br J Cancer 2017 May 9;116(10):1264-70.

13. Systemic Therapy and chemoradiation in advanced localised pancreatic cancer - 2 (SCALOP-2). [Available at: https://clinicaltrials.gov/ct2/show/ NCT02024009]

14. Radiation therapy with cisplatin or cetuximab in treating patients with oropharyngeal cancer. [Available at: https://clinicaltrials.gov/ct2/show/NC To1302834?term =RTOG1016\&rank=1]

15. Lee JH, Kang CM, Bang SM, et al. The role of neoadjuvant chemoradiation therapy in patients With borderline resectable pancreatic cancer with isolated venous vascular involvement. Medicine (Baltimore) 2015 Aug;94(31):e1233.

16. Gillen S, Schuster T, Meyer Zum Büschenfelde C, Friess H, Kleeff J. Preoperative/neoadjuvant therapy in pancreatic cancer: A systematic review and metaanalysis of response and resection percentages. PLoS Med 2010 Apr 20;7(4):e1000267.

17. Krishnan S, Chadha AS, Suh Y, et al. Focal radiation therapy dose escalation improves overall survival in locally advanced pancreatic cancer patients receiving induction chemotherapy and consolidative chemoradiation. Int J Radiat Oncol Biol Phys 2016 Mar 15;94(4):755-65.

18. Heerkens HD, van Vulpen M, van den Berg CA, et al. MRI-based tumor motion characterization and gating schemes for radiation therapy of pancreatic cancer. Radiother Oncol 2014 May;111(2):252-7.

19. Fokas E, Spezi E, Patel N, et al. Comparison of investigator-delineated gross tumour volumes and quality assurance in pancreatic cancer: Analysis of the on-trial cases for the SCALOP trial. Radiother Oncol 2016 Aug;120(2):212-6.

20. Li XX, Liu NB, Zhu L, et al. Consequences of additional use of contrast-enhanced (18)F-FDG $\mathrm{PET} / \mathrm{CT}$ in target volume delineation and dose distribution for pancreatic cancer. Br J Radiol 2015 Jul;88(1051):20140590. 


\section{EDORiUM Journals}

www.ijhpd.com

21. Wilson JM, Mukherjee S, Brunner TB, Partridge M, Hawkins MA. Correlation of 18 F-fluorodeoxyglucose positron emission tomography parameters with patterns of disease progression in locally advanced pancreatic cancer after definitive chemoradiotherapy. Clin Oncol (R Coll Radiol) 2017 Jun;29(6):370-7.

22. Mahadevan A, Jain S, Goldstein M, et al. Stereotactic body radiotherapy and gemcitabine for locally advanced pancreatic cancer. Int $J$ Radiat Oncol Biol Phys 2010 Nov 1;78(3):735-42.

23. Moningi S, Dholakia AS, Raman SP, et al. The role of stereotactic body radiation therapy for pancreatic cancer: A single-institution experience. Ann Surg Oncol 2015 Jul;22(7):2352-8.

$* * * * * * * * *$

\section{Author Contributions}

Sophie Otter - Substantial contributions to conception and design, Acquisition of data, Analysis and interpretation of data, Drafting the article, Revising it critically for important intellectual content, Final approval of the version to be published

Irene Chong - Substantial contributions to conception and design, Acquisition of data, Analysis and interpretation of data, Drafting the article, Revising it critically for important intellectual content, Final approval of the version to be published
Ria Kalaitzaki - Substantial contributions to conception and design, Acquisition of data, Analysis and interpretation of data, Drafting the article, Revising it critically for important intellectual content, Final approval of the version to be published

Diana Tait - Substantial contributions to conception and design, Acquisition of data, Analysis and interpretation of data, Drafting the article, Revising it critically for important intellectual content, Final approval of the version to be published

\section{Guarantor of Submission}

The corresponding author is the guarantor of submission.

\section{Source of Support \\ None}

\section{Conflict of Interest}

Authors declare no conflict of interest.

\section{Copyright}

(C) 2018 Sophie Otter et al. This article is distributed under the terms of Creative Commons Attribution License which permits unrestricted use, distribution and reproduction in any medium provided the original author(s) and original publisher are properly credited. Please see the copyright policy on the journal website for more information.
Access full text article on other devices

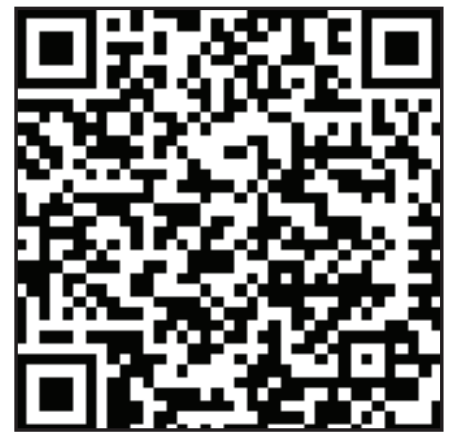

Access PDF of article on other devices

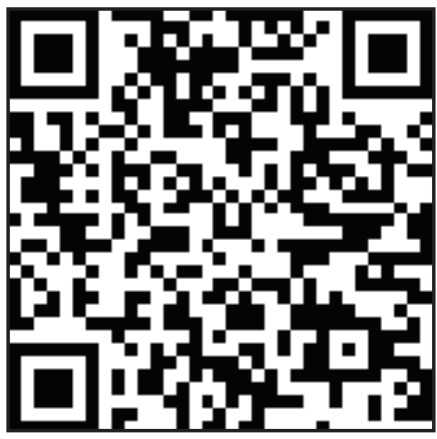

